\title{
Hemolytic Anemia due to Membrane Defect
}

National Cancer Institute

\section{Source}

National Cancer Institute. Hemolytic Anemia due to Membrane Defect. NCI Thesaurus.

Code C101218.

A group of inherited hemolytic anemias caused by erythrocyte membrane defects. This includes hereditary pyropoikilocytosis, hereditary spherocytosis and hereditary elliptocytosis. 